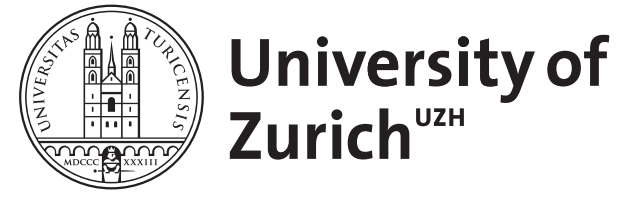
Archive

University of Zurich

University Library

Strickhofstrasse 39

CH-8057 Zurich

www.zora.uzh.ch

Year: 2003

\title{
Die Pflichten des Skeptikers : Eine Kritik an Peter Stemmers moralischem Kontraktualismus
}

Schaber, Peter

Posted at the Zurich Open Repository and Archive, University of Zurich

ZORA URL: https://doi.org/10.5167/uzh-80624

Book Section

Published Version

Originally published at:

Schaber, Peter (2003). Die Pflichten des Skeptikers : Eine Kritik an Peter Stemmers moralischem Kontraktualismus. In: Leist, Anton. Moral als Vertrag? Beiträge zum moralischen Kontraktualismus. Berlin: de Gruyter, 199-212. 


\title{
Die Pflichten des Skeptikers. Eine Kritik an Peter Stemmers moralischem Kontraktualismus*
}

\author{
Peter Schaber
}

Es gehört zur Grundidee des moralischen Kontraktualismus, moralische Normen als Normen zu verstehen, auf die sich Menschen im Lichte ihres Ziels, ihre Interessen optimal zu befriedigen, einigen könnten. Dabei ist das Motiv der Zustimmung zu Regeln des Zusammenlebens nichts anderes als genau dieses Ziel, ihre Interessen optimal zu befriedigen. Der moralische Kontraktualismus liefert uns entsprechend auch eine Antwort auf die Frage, was es denn sei, das uns auf die Befolgung der Regeln, auf die wir uns einigen würden, verpflichtet: im Kern nichts anderes als unser Eigeninteresse.

Der Gedanke, dass unser Eigeninteresse uns verpflichtet, ist erläuterungsbedürftig. Es ist nämlich bloss dann in unserem Eigeninteresse, die moralischen Normen, die wir selber in der fiktiven Situation des Vertragsschlusses befürwortet hätten, auch zu befolgen, wenn bestimmte Bedingungen erfüllt sind. Die Regelbefolgung entspricht genau dann meinem Eigeninteresse, wenn ich vernünftigerweise davon ausgehen kann, dass eine Regelverletzung für mich negative Konsequenzen nach sich ziehen wird. Und dies ist mit Sicherheit dann der Fall, wenn die Verletzung einer Regel mit Sanktionen verbunden ist.

In diesen Sanktionen sieht der moralische Kontraktualist Peter Stemmer den Schlüssel zur Idee des moralischen Müssens. Was uns moralisch verpflichtet, die Regeln auch zu befolgen, auf die wir uns in der fiktiven Situation des Vertragsschlusses einigen würden - dies der Kern von Stemmers Vorschlag - ist nichts anders als die Sanktion, die uns trifft, wenn wir die Regel nicht befolgen. Stemmer glaubt damit einen Begriff moralischer Verpflichtung zu formulieren, der nicht zurückgreifen muss, auf, wie er meint, „mysteriöse Kräfte" 1 wie objektive Werte und objektive Normen. Hier liegt nach Stemmer vielmehr eine metaphysikfreie, aufgeklärte Moral vor, eine Moral, die keine dubiosen ontologischen Verpflichtungen eingeht. ${ }^{2}$ Mit diesem Konzept mora-

\footnotetext{
* Für wertvolle Hinweise möchte ich Anton Leist, Neil Roughley, Thomas Schmidt, Gottfried Seebaß und Peter Stemmer danken.

1 Stemmer (2000) 114.
} 
lischer Verpflichtung werde ich mich nachfolgend beschäftigen. Dabei möchte ich auf einige Schwierigkeiten hinweisen, die es nahelegen, Stemmers Konzept zurückzuweisen.

\section{Stemmers Idee}

Betrachten wir Stemmers Vorschlag etwas genauer. Stemmer bezeichnet das moralische Müssen, das er ins Zentrum seiner Ausführungen stellt, in Übereinstimmung mit Ernst Tugendhat als ein „sanktionsbedingtes Müssen“. ${ }^{3}$ Ein Versprechen z.B. muss ich halten, sofern ich die Sanktion vermeiden will, die über mich verhängt wird, wenn ich mein Versprechen nicht halte. Dabei handelt es sich beim moralischen Müssen nach Stemmer um ein selbstauferlegtes Müssen. ${ }^{4}$ Dass meine Verletzung einer Norm sanktioniert wird, beruht auf meinem Einverständnis, das ich im Lichte meiner Eigeninteressen gegeben habe bzw. geben würde, betrachtete ich die jeweils zur Diskussion stehende Norm im Lichte meines Eigeninteresses. Das heisst nicht, dass ich in der Handlungssituation, in der die Sanktion verhängt wird, einen Wunsch habe, dass dies auch geschehe. Im Gegenteil. Es ist davon auszugehen, dass ich keinen entsprechenden Wunsch habe. Trotzdem ist das moralische Müssen, wie Stemmer meint, ein selbstauferlegtes Müssen: „Das moralische Müssen ist gewollt, und dennoch ist es ein echtes Müssen, weil das einmal etablierte Sanktionensystem die Sanktionen verhängt, ohne dass der einzelne, der zusammen mit anderen dieses System geschaffen hat, daran etwas ändern kann. ${ }^{\circ}$

Nicht jede künstlich geschaffene Sanktion bringt nun aber nach Stemmer ein moralisches Müssen hervor. Das ist seiner Ansicht nach deshalb nicht der Fall, weil sonst die Sanktion, mit der ein Bankräuber z.B. einem Bankangestellten droht, ein moralisches Müssen erzeugen würde. Wäre jede künstlich geschaffene Sanktion moralisch verpflichtend, wäre es die moralische Pflicht des Bankangestellten, das geforderte Geld dem Bankräuber zu übergeben. Nach Stemmer folgt dies aber nicht aus seiner Konzeption, da ein moralisches Müssen nur durch Sanktionen hervorgebracht wird, welche durch diejenigen geschaffen wurden, die dazu vorgängig autorisiert worden sind. ${ }^{6}$ Das heisst: Moralische Verpflichtungen werden nach Stemmer nur dann durch Sank-

\footnotetext{
2 Ebd. 114.

3 Ebd. 102.

4 Anders als für Tugendhat ist für Stemmer bloss die selbstauferlegte und entsprechend künstlich geschaffene Sanktion für das moralische Müssen relevant; vgl. dazu ebd. 103.

5 Ebd. 110.

6 Ebd. 114.
} 
tionen generiert, wenn die Sanktionsinstanz berechtigt ist, die Sanktionen zu verhängen. Das unterscheidet, wie Stemmer meint, das moralische vom erpresserischen Müssen. Und dementsprechend gilt: „Was moralisch verpflichtet, sind nicht irgendwelche mysteriösen Kräfte, sondern Sanktionen, aber Sanktionen, die eine Instanz verhängt, die dazu berechtigt ist. " ${ }^{7}$ Dabei besteht diese Instanz aus denjenigen, die zugleich die Adressaten der moralischen Verpflichtung sind. ${ }^{8}$

Soweit Stemmers Vorschlag. Sollten wir diesen Vorschlag akzeptieren? Ich werde nachfolgend einige Gründe anführen, wieso wir das nicht tun sollten. Dabei möchte ich zeigen, dass Stemmer nicht über die theoretischen Ressourcen verfügt, einen Begriff moralischer Verpflichtung zu formulieren (2.), sich aus Stemmers Konzept kein Kernbereich allgemein geltender moralischer Pflichten herleiten lässt (3./4.) und die wunschrelativen Handlungsgründe, die Stemmer für die einzigen Handlungsgründe hält, ein wunschunabhängiges Sollen voraussetzen (5.).

\section{Das moralische Müssen als prudentielles Müssen}

Für Stemmer ist das moralische Müssen eine Art des prudentiellen Müssens. ${ }^{9}$ Trotzdem unterscheidet er das moralische von einem bloss prudentiellen Müssen. Betrachten wir ein Beispiel eines prudentiellen Müssens: Ich muss Gymnastik machen, wenn ich gesund werden will. Dieses prudentielle Müssen unterscheidet sich nach Stemmer von einem moralischen Müssen („Ich muss meine Versprechen halten ") in zwei Hinsichten: a) es ist nicht durch Menschen geschaffen und b) es verdankt sich keinem Handeln, zu dem die Handelnden autorisiert worden $\operatorname{sind}^{10}:$ „Nichts von alledem findet sich in der Situation, in

7 Ebd. 114.

8 Vgl. ebd. 115: „Die Autoren der verpflichtenden Ordnung sind zugleich auch deren Adressaten. “ In seinem neueren Aufsatz ,Moralischer Kontraktualismus" hat Stemmer dieses Konzept des moralischen Müssen leicht revidiert. Danach liegt ein moralisches Müssen nur dann vor, wenn eine Forderung neben der Verbindung mit einer Sanktion zugleich auch die Eigenschaft aufweist, dem Interesse der von der Forderung Betroffenen dienlich zu sein; vgl. Stemmer (2002) 15: „Welche Eigenschaft ist es nun genau, die einer moralischen Norm Legitimität und verpflichtenden Charakter verleiht? ... Der Norm muss, so die Antwort, eine bestimmte Interessenkonfiguration, eine bestimmte Interessenlage bei den Betroffenen zugrunde liegen ... Die Legitimität der Norm resultiert aus der Eigenschaft, einer Interessenkonstellation ... zu entsprechen."

9 Vgl. Stemmer (2000) 118: „Das moralische Müssen ist ... ein besonderes prudentielles Müssen, es steht als eine besondere Art dieses Müssens neben dem bloss prudentiellen Müssen.“

10 Vgl. ebd. 118f.: „Das moralische Müssen ... ist ein durch Sanktionen künstlich geschaffenes Müssen. Es ist zum zweiten ein Müssen, das nicht durch gewalttätiges, erpresserisches Handeln entsteht, sondern durch berechtigtes Handeln. Mitglieder der moralischen Gemeinschaft 
der sich jemand einer Gymnastik unterziehen muss, wenn er wieder gesund werden will, und ein anderer ihm deshalb rät, sich für die Gymnastik zu entscheiden." ${ }^{11}$ Die negativen Konsequenzen, die drohen, wenn die betroffene Person keine Gymnastik betreibt, werden nicht von anderen Menschen verhängt und ergeben sich insofern auch nicht aus autorisierten Sanktionen.

Doch auch wenn das zutrifft, bleibt unklar, ob das moralische Müssen wirklich eine - wie Stemmer sagt - besondere Art prudentiellen Müssens ist. Dies bleibt deshalb unklar, weil das Müssen in beiden Fällen - im prudentiellen wie im moralischen - auf dem Wollen der betroffenen Person beruht. Paul muss Gymnastik machen, sofern er gesund werden will. In gleicher Weise gilt: Paul muss das gegebene Versprechen halten, sofern er die ihm drohende Sanktion vermeiden will. Würde er das gegebenenfalls nicht wollen, wäre die von der moralischen Gemeinschaft verhängte Sanktion normativ gesehen für ihn nicht von Belang (genauso wie er keine Gymnastik treiben müsste, wenn er gegebenenfalls nicht gesund werden möchte). Das heisst, dass die von Stemmer genannten Merkmale, die das moralische Müssen als ein besonderes prudentielles Müssen auszeichnen sollen, nicht konstitutiv sind für das moralische Müssen. Deshalb lässt sich das moralische Müssen über diese Merkmale in normativer Hinsicht in keiner Weise vom prudentiellen Müssen unterscheiden (es wird dadurch nicht zu einer - wie Stemmer meint - besonderen Art des prudentiellen Müssens). Das Müssen, von dem im Gymnastikbeispiel die Rede ist, ist genauso wollensbedingt wie das Müssen, das in der Aussage „Paul muss sein Versprechen halten“ zum Ausdruck gebracht wird.

Das heisst: Auf die Frage eines Skeptikers, wieso er denn seine Versprechen halten müsse, lautet die Antwort gleich wie im Gymnastikbeispiel: Du musst das tun, weil es in deinem Interesse ist. ${ }^{12}$ Deshalb sind die von Stemmer genannten Merkmale, die das moralische vom bloss prudentiellen Müssen unterscheiden, nichts, was die beiden Müssen in normativer Hinsicht unterscheiden würden. Das moralische Müssen mag durch Menschen geschaffen sein und sich berechtigtem Handeln verdanken; das ändert nichts daran, dass die Frage „Wieso muss ich?“ in beiden Fällen dieselbe Antwort hat.

Nun könnte man dies aus Stemmers Sicht nicht für weiter problematisch halten. Seiner Meinung nach ist das moralische Müssen ein prudentielles Müssen, so dass er mit dem obigen Befund durchaus gut leben kann. Das Problem allerdings liegt darin, dass man im Zusammenhang mit dem moralischen

haben den moralischen Raum selbst geschaffen, sie haben die moralischen Sanktionen selbst gewollt und die Sanktionsinstanz autorisiert, die Sanktionen ... zu verhängen.“

11 Ebd. 119.

12 Dies entspricht auch Stemmers Idee der Begründung einer Handlung; vgl. ebd. 37: „Eine Handlung zu begründen, heisst, zu zeigen, dass, so zu handeln, im Interesse des Handelnden liegt ..." 
Müssen nicht mehr sinnvoll von Verpflichtung reden kann. Das heisst: Es gibt dann nichts mehr, wozu man verpflichtet ist. Dort, wo ein prudentielles Müssen vorliegt, spricht man nämlich nicht von Verpflichtung. Paul ist nicht verpflichtet, Gymnastik zu machen. Natürlich ist es in seinem Interesse, dies zu tun. Und man kann ihm deshalb auch den Rat geben, sich entsprechend zu verhalten. Aber wir werden ihm keine Vorwürfe machen, wenn er es nicht tut. Wir wären auch nicht empört darüber, wenn er untätig bliebe. Es wäre unklug von ihm, sich so zu entscheiden. Auf die möglicherweise schmerzhafte Gymnastik zu verzichten, wäre aber nicht moralisch falsch, weil Paul damit keine Pflicht verletzt. Wenn nun aber - und darin liegt das Problem - das moralische Müssen nichts anderes ist als ein prudentielles Müssen, dann gilt dasselbe auch für das moralische Müssen. Dies hat zur Folge, dass man nicht mehr sinnvoll von moralischen Verpflichtungen reden kann. Es ist im Interesse von Paul, sein Versprechen zu halten, wenn ihm eine Sanktion droht. Es wäre entsprechend auch unklug von ihm, das Versprechen nicht zu halten. Wir hätten aber keinen Grund, ihm Vorwürfe zu machen. Vorwürfe können wir ihm sinnvollerwiese nur dann machen, wenn er etwas nicht getan hat, wozu er verpflichtet war. Dies ist aber, wenn das moralische Müssen ein prudentielles Müssen ist, nie der Fall.

Mit diesem Befund kann Stemmer aber nicht gut leben. Es widerspricht zum einen seinem Verständnis des sanktionstheoretischen Ansatzes des moralischen Müssens: „Auf dem Boden dieser Konzeption wird erst klar, was es heisst, eine Verpflichtung, ein Recht zu haben, und was es heisst, eine Verpflichtung zu übernehmen und ein Recht zuzuerkennen. Und damit wird erst verständlich, was die Basis für eine moralische Forderung ist. "13 Zum anderen wäre eine Analyse moralischen Müssens, in der die Rede von Verpflichtung keinen Sinn mehr machen würde, doch sehr sonderbar.

Dieser Schluss würde nicht folgen, wenn man es als gefordert ansehen würde, seine eigenen Interessen zu verfolgen. Auch das prudentielle Müssen wäre dann ein verpflichtendes Müssen. Verpflichtend wären dabei die negativen Konsequenzen, die sich aus der Vernachlässigung der eigenen Interessen ergeben würden.

Stemmer steht diese Option nicht offen. Er versteht „Sanktion“ explizit - gegen Tugendhat - nicht im Sinne von negativer Konsequenz. ${ }^{14}$ Zudem wäre auch unklar, inwiefern negative Konsequenzen verpflichtend wären, sofern es

13 Ebd. 119.

14 Ebd. 103: Bei Tugendhat ist - so Stemmer - „das praktische Müssen insgesamt sanktionskonstituiert. Damit ist die These über das moralische Müssen wesentlich unspezifischer, sie besagt nur, dass wie für das praktische Müssen insgesamt auch für das moralische Müssen gilt, dass, wer anders als ,gemusst' handelt, mit einer negativen Konsequenz zu rechnen hat.“ 
sich dabei um etwas handelt, das der Handelnde nicht vermeiden will. Was aber soll uns sonst - wenn es die negativen Konsequenzen nicht tun - auf die Verfolgung der eigenen Interessen verpflichten? Für jemanden, der wie Stemmer der Ansicht ist, dass das moralische Müssen nicht von den Interessen der Handelnden loszulösen ist, kann die Antwort hier nur lauten: nichts. Deshalb darf man mit guten Gründen auch an der oben gegebenen Diagnose festhalten: Es fehlen in Stemmers Analyse des moralischen Müssens die theoretischen Ressourcen für einen Begriff der Verpflichtung.

\section{Ein universaler Kernbereich moralischer Pflichten?}

Doch auch wenn Stemmer das moralische Müssen als eine besondere Art des prudentiellen Müssens auszeichnen könnte, wäre sein Konzept des moralischen Müssens aus einem anderen Grund zurückzuweisen. Anders als Stemmer selbst glaubt, lässt sich mit seinem Konzept kein Kernbereich moralischer Pflichten formulieren. ${ }^{15}$ Die Überlegung Stemmers, die ich hier im Blick habe, ist folgende.

Das Ziel Stemmers ist es, Moral in einer Weise zu begründen, die auch für den moralischen Skeptiker, der unter anderem dadurch charakterisiert ist, dass er kein Interesse an den Interessen anderer hat, akzeptabel ist. ${ }^{16}$ Dies ist nach Stemmer der Prüfstein einer universalen Moral. Denn das, was man dem moralischen Skeptiker gegenüber begründen kann, hat für alle Geltung. Was für denjenigen, der den Anliegen der anderen indifferent gegenübersteht, als Verpflichtungsgrund akzeptabel ist, wird für den Altruisten schon gar nicht in Frage stehen. Deshalb meint Stemmer: „Der Kontraktualismus in der hier entwickelten Form entwirft eine Moral, an der teilzuhaben für jeden und damit auch für den moralischen Skeptiker vernünftig ist. Diese Moral ist die eine für alle vernünftige Moral mit dem einen vernünftigen Inhalt."17

Ihre Grundlage hat diese vernünftige Moral nach Stemmer in den basalen Interessen der Menschen, in den Interessen, die von allen geteilt werden. Jeder von uns hat ein Interesse daran, nicht getötet, nicht physisch oder psychisch verletzt, nicht erniedrigt, nicht bestohlen zu werden. Dementsprechend haben alle - moralische Skeptiker in gleicher Weise wie Altruisten - ein Interesse daran, dass moralische Normen wie „Du sollst nicht töten“, „Du sollst den

15 Stemmer selbst redet in diesem Zusammenhang von einem „Kernbestand von elementaren Rechten und Pflichten“ bzw. von einer „Minimalmoral“, vgl. ebd. 292.

16 Die fiktive Figur des moralischen Skeptikers zeichnet sich darüberhinaus dadurch aus, dass sie keinen moralischen Standpunkt einnimmt, keine religiöse Überzeugungen besitzt und auch nicht an die Existenz einer objektiven normativen Welt glaubt; vgl. dazu ebd. 18f.

17 Ebd. 209. 
anderen nicht verletzen“ etc. allgemeine Geltung haben. So lassen sich auch einige zentrale Pflichten dieser vernünftigen Minimalmoral formulieren: „die Pflicht, nicht zu töten, die Pflicht, dem anderen im Falle einer Lebensgefahr zu helfen, die Pflicht, den anderen körperlich nicht zu verletzen. Weiter die Pflicht, den anderen in der Entfaltung und im Gebrauch seiner geistigen Fähigkeiten nicht zu behindern, die Pflicht, ihn psychisch nicht zu schädigen, und schliesslich die Pflicht, ihn nicht zu demütigen und zu erniedrigen. " ${ }^{18}$ Das sind die moralischen Pflichten, zu deren Einhaltung, Anerkennung und Befolgung alle guten Grund haben. Dieser Kernbestand moralischer Pflichten verbietet es nach Stemmer natürlich niemandem, sich um andere über das Mass hinaus, das durch diese Minimalpflichten vorgeben wird, zu kümmern. Neben den moralischen Pflichten gibt es auch altruistische Ideale, nach denen zu streben, Stemmer zufolge natürlich jedem freisteht. ${ }^{19}$ Was Ideale von Pflichten unterscheidet, ist dies, dass sie nicht von allen eingefordert werden können. Wer nicht die altruistischen Interessen hat, welche diesen Idealen zugrunde liegen, hat auch keinen Grund, ihnen gemäss zu handeln. Dem moralischen Skeptiker gegenüber lassen sie sich entsprechend auch nicht begründen.

Formuliert Stemmers Kontraktualismus also einen Kernbereich moralischer Normen, die universale Geltung besitzen? Gibt es - anders gefragt - einen Bestand von Verpflichtungen, die wir alle teilen? Ich glaube, dass Stemmers Vorschlag hier mit einer Schwierigkeit konfrontiert ist, die sämtliche kontraktualistische Moraltheorien teilen: Er kann zeigen, dass alle Menschen gute Gründe haben, gewisse moralische Normen anzuerkennen, nicht aber, dass sie auch gute Gründe haben, diese Normen auch zu befolgen. Wenn das richtig ist, dann formuliert Stemmer keinen Kernbestand von moralischen Pflichten, die eine allgemeine Geltung haben. Dabei verstehe ich unter einer Pflicht, die eine allgemeine Geltung hat, eine Pflicht, die für alle Handelnden in allen Situationen, in denen die Pflicht befolgt werden kann, verbindlich ist, sofern sie nicht mit anderen, gewichtigeren moralischen Pflichten kollidiert. Die genauere Betrachtung zeigt nun aber, dass Stemmer solche moralischen Pflichten nicht zu begründen vermag.

Erinnern wir uns: Was uns nach Stemmer moralisch verpflichtet, sind die Sanktionen der moralischen Gemeinschaft, die dazu autorisiert ist, diese Sanktionen zu verfügen. Ich muss einer Person, die in Lebensgefahr ist, helfen, weil ich sonst eine Sanktion erfahren würde. Nun kann man realistischerweise nicht davon ausgehen, dass jede Normverletzung sanktioniert wird. Auch wenn jede Normverletzung, die entdeckt wird, sanktioniert wird, ist nicht davon auszugehen, dass jede Normverletzung eine Sanktion nach sich ziehen wird: Wir leben nicht in einer Welt, in der eine entsprechende Überwachung

18 Ebd. 209f.

19 Vgl. dazu ausführlich ebd. 291ff. 
möglich ist. ${ }^{20}$ Wenn es nun aber die berechtigte Sanktion ist, die uns moralisch verpflichtet, dann sind wir in den Situationen, in denen wir nicht mit einer Sanktion zu rechnen haben, auch in keiner Weise verpflichtet, die Norm - auf die wir uns im Lichte unserer basalen Interessen geeinigt hätten - zu befolgen. Jeder hat ein Interesse an der allgemeinen Geltung der von Stemmer genannten moralischen Normen. Gleichzeitig aber haben nicht alle auch in jedem Fall ein Interesse daran, die Norm zu befolgen. Ob ich verpflichtet bin, die Normen zu befolgen, hängt davon ab, ob ich mit einer Sanktion rechnen kann oder nicht. Deshalb kann man nicht sagen, dass wir alle verpflichtet seien, die Stemmerschen Normen auch zu befolgen. Obwohl wir in der Vertragssituation eine Norm unterschreiben würden, die besagt, dass wir alle verpflichtet sind, die Norm ,Du sollst den anderen nicht bestehlen' einzuhalten, wären die einzelnen in verschiedenen Situation nicht dazu verpflichtet, diese Norm auch wirklich zu befolgen. Der Inhalt der Minimalmoral Stemmers hängt davon ab, ob eine Normbefolgung im Eigeninteresse des Handelnden ist oder nicht: Während ich verpflichtet sein kann, meinen Nachbarn nicht zu bestehlen, kann mein Freund gleichzeitig nicht verpflichtet sein, dies auch seinem Nachbarn gegenüber zu unterlassen. Wir unterstehen deshalb nicht notwendigerweise denselben Pflichten. Die Pflicht, den anderen z.B. nicht zu betrügen, hat nicht in allen Situationen, in denen sie befolgt werden kann und in der sie nicht mit einer gewichtigeren moralischen Pflichten kollidiert, Geltung.

\section{Innere Sanktionen und eine Disposition zur Moral}

Stemmer sieht dieses Problem und versucht es auf folgende Weise zu lösen: Er glaubt, dass dieses „Unrechttun im Verborgenen “ 21 auf wenige Fälle reduziert werden kann, weil der Handelnde auch in diesen Fällen mit einem „Bündel von Nachteilen “22 zu rechnen hätte. Stemmer nennt drei Gründe, die gegen unmoralisches Handeln im Verborgenen sprechen:

a) Zunächst könne man in vielen Fällen nicht ausschliessen, danach doch noch entdeckt und entsprechend dann auch sanktioniert zu werden. Deshalb sei es vernünftig, auch in diesen Situationen sich moralisch zu verhalten. Das ist richtig; allerdings lässt sich auch nicht bestreiten, dass in vielen Fällen kaum davon auszugehen ist, dass die Normverletzung entdeckt wird. Und das spricht in den entsprechenden Situationen für ein Unrechttun im Verborgenen.

20 Zudem wäre sie, wenn sie möglich wäre, wohl auch nicht wünschenswert.

$21 \mathrm{Vgl}$. dazu ebd. $162 \mathrm{ff}$.

22 Ebd. 190. 
b) Gegen ein Unrechttun im Verborgenen sprechen die inneren Sanktionen, denen man sich nach Stemmer nicht entziehen kann. Es geht dabei um eine innere Ablehnung des Unrechttuns, ein inneres Unbehagen gegenüber unmoralischem Verhalten, das, so Stemmer, deshalb unvermeidlich ist, da Menschen „gar nicht anders können, als das, was sie tun oder tun wollen, auch aus der Perspektive der anderen zu betrachten. "23 Das spricht gegen unmoralisches Handeln auch in Situationen, in denen mit einer äusseren Sanktion nicht gerechnet werden muss. Da sich niemand, auch der moralische Skeptiker nicht, den inneren Sanktionen entziehen kann, haben alle Grund, sich auch im Verborgenen moralisch zu verhalten. Nach Stemmer ist dieser Grund nicht bei allen gleich stark. Für jemanden wie den moralischen Skeptiker ist dieses Müssen - anders als für Menschen mit altruistischen Präferenzen - „nicht sehr stark“. Und dies hat zur Konsequenz, dass ,auch das moralische Müssen, das durch diese Sanktionen konstituiert wird, nicht sehr stark “24 ist.

Doch unabhängig davon, wie stark das entsprechende Müssen ist, ${ }^{25}$ die inneren Sanktionen tragen zur Lösung des vorliegenden Problems nichts bei. Und dies aus folgendem Grund: Innere Sanktionen sind in Stemmers ,aufgeklärter' Moral irrationale Grössen. Wenn der moralische Skeptiker rational ist, wird er keine innere Sanktionen erfahren. Wieso soll jemand wie der moralische Skeptiker, der kein Interesse hat an den Interessen anderer Menschen, sich Vorwürfe machen oder ein inneres Unbehagen empfinden, wenn er gegen die Interessen der anderen handelt? Wenn das, was er dabei tut, in seinem Interesse ist, handelt er genauso wie er handeln muss. Er hat sich entsprechend auch nichts vorzuwerfen. Wenn jemand wie der moralische Skeptiker dennoch bei solchen Handlungen ein inneres Unbehagen spürt, ist er Opfer von Verpflichtungsideen, die nicht Bestandteil der von Stemmer intendierten aufgeklärten Moral sein können. Wenn sich der moralische Skeptiker dies klar macht, wird sich das innere Unbehagen bei Normverletzungen auch nicht mehr einstellen. Das Unbehagen beruht nämlich auf der irrigen Vorstellung, etwas falsch gemacht zu haben. Wie aber die aufgeklärte Moral lehrt, hat er nichts falsch gemacht. Im Gegenteil. Er hat so gehandelt, wie er handeln musste. Wer sich dies vergegenwärtigt, befreit sich von jedem inneren Unbehagen, das sich im Zusammenhang von Normverletzungen, die in seinem Interesse sind, einstellen könnten.

23 Ebd. 173.

24 Ebd. 189.

25 Es ist unklar, ob die Rede von Graden des Müssens überhaupt sinnvoll ist. Das praktische Müssen ganz allgemein scheint eine Alles-oder-Nichts-Angelegenheit zu sein. Ich muss meinem Nachbarn helfen oder ich muss das nicht tun. Es macht wenig Sinn, zu sagen, dass ich dies ein wenig oder sehr stark tun muss. 
c) Nach Stemmer spricht noch ein dritter Punkt gegen das Unrechttun im Verborgenen: Die genauere Betrachtung zeigt, wie Stemmer glaubt, dass es für jeden vernünftig ist, eine Disposition auszubilden, moralisch zu handeln. Personen, die nicht zur Moral disponiert sind, werden - wenn sie entdeckt werden - von der moralischen Gemeinschaft sanktioniert. Dies deshalb, weil von diesen Personen zu erwarten ist, dass sie unmoralisch handeln, wenn das für sie von Vorteil ist. Diese - so Stemmer - sekundären Sanktionen verwandeln sich in innere Sanktionen: Das Abweichen von der Moral löst ein inneres Unbehagen aus. Und nach Stemmer folgt daraus: „Die Mitglieder der moralischen Gemeinschaft sind nicht nur zu Handlungen, sondern auch zu Dispositionen zu Handlungen moralisch verpflichtet. “26

Dies folgt natürlich bloss dort, wo wir, wie Stemmer selbst zugibt, mit anderen nahe zusammenleben und entsprechend in der Lage sind, die Dispositionen der anderen zu erkennen. ${ }^{27}$ Doch unabhängig davon, stellt sich die Frage, ob es für jemanden wie den moralischen Skeptiker rational ist, eine Disposition auszubilden, moralisch zu handeln. Zunächst ist klar, dass dies nur dann rational sein kann, wenn auch die anderen bereit sind, dies zu tun. ${ }^{28}$

Allerdings zeigt die nähere Betrachtung, dass es für den Skeptiker auch dann, wenn diese Bedingung erfüllt ist, nicht rational ist, diese Disposition auszubilden. Wer eine stabile Disposition ausbildet, moralisch zu handeln, um sekundäre Sanktionen zu vermeiden, würde auch in den Situationen moralisch handeln, in denen es für ihn nicht vorteilhaft wäre. Im Eigeninteresse des moralischen Skeptikers wäre es aber, in seinem Handeln flexibel zu bleiben und in den Situationen moralisch zu handeln, in denen das für ihn von Vorteil ist, und in den Situationen nicht moralisch zu handeln, in denen das nicht der Fall ist. Und entsprechend wäre es unvernünftig, eine allgemeine Disposition auszubilden, moralisch zu handeln. Aus diesem Grund kann man auch nicht auf eine solche Disposition hoffen, um das Problem des Unrechttuns im Verborgenen zu lösen. Das Problem der Normenbefolgung bleibt vielmehr ungelöst und dies ist, wie Stemmer selbst an einer Stelle schreibt, „für die am Projekt der Moral Beteiligten, auch für den moralischen Skeptiker, unbefriedigend“. ${ }^{29}$

26 Ebd. 188.

27 Ebd. 188.

28 Vgl. dazu ebd. 182: „Die Ausbildung der eigenen Disposition ist ... die notwendige Voraussetzung für die Ausbildung der Disposition des anderen, und genauso umgekehrt.“

29 Ebd. 186. 


\section{Mysteriöse Grössen?}

Dass das Problem der Normenbefolgung nicht so, wie die am Projekt der Moral Beteiligten das wünschen, gelöst werden kann, betrachtet Stemmer als eine Schwäche des Kontraktualismus. Wie er aber hinzufügt, handelt es sich dabei um eine „unvermeidliche Schwäche“. ${ }^{30}$ Unvermeidlich deshalb, weil ein umfassenderer Begriff der moralischen Verpflichtung und des moralischen Müssens innerhalb einer aufgeklärten Moral, in der nicht auf objektive Werte und objektive Normen verwiesen werden kann, keinen Platz hat. Moralische Normen können in einer aufgeklärten Moral nur über die Interessen der Handelnden begründet werden. Einen Grund, eine Norm zu befolgen, habe ich nach Stemmer dann und nur dann, wenn die geforderte Normbefolgung in meinem Interesse ist bzw. einem meiner Wünsche dienlich ist. Für Stemmer gibt es keine Handlungsgründe ohne Wünsche. „Die Wünsche sind ... das, woran sich entscheidet, was zu tun vernünftig ist ... Vernünftig sind die Handlungen, die man tun muss, um seine Wünsche optimal zu erfüllen. “31 Deshalb kann eine Handlung auch nur dann als vernünftig oder rational zwingend ausgewiesen werden, wenn man zu zeigen in der Lage ist, dass sie einem oder mehreren Wünschen des Handelnden förderlich ist. Dies scheint nun im Blick auf die Gründe, moralische Normen zu befolgen, wie wir gesehen haben, unbefriedigende Konsequenzen nach sich zu ziehen: Eine aufgeklärte Moral muss diesen Preis bezahlen, was nach Stemmer ohne Zweifel weniger schwer wiegt als die Zulassung von mysteriösen Grössen, wie z.B. objektiven Werten.

Allerdings denke ich, dass diese Grössen weniger mysteriös sind, als Stemmer glaubt. Und nicht nur dies: Die nähere Betrachtung zeigt, dass die wunschrelativen Handlungsgründe, von denen Stemmer redet, Gründe zum Handeln voraussetzt, die wunschunabhängig sind. Das lässt mit Hilfe der folgenden Überlegung deutlich machen.

Stemmer hält Gründe, die auf keinen Wünschen beruhen, für mysteriöse Grössen. Es sind nach Stemmer einzig Wünsche, die uns mit Handlungsgründen versorgen. Und zwar liefern sie uns Gründe, diejenigen Handlungen auszuführen, welche die geeigneten Mittel zur Befriedigung unsere Wünsche darstellen. Handlungsgründe ergeben sich nach dieser Vorstellung ausschliesslich als Schluss folgender Prämissen: ${ }^{32}$

1. Ich wünsche A.

2. $\mathrm{Y}$ ist das geeignete Mittel für A.

3. Ich habe einen Grund, Y zu wählen.

30 Ebd. 190.

31 Ebd. 21.

32 Vgl. dazu Korsgaard (1997); auch Nagel (1999) und Schaber (1999). 
An diesem Schluss fällt auf, dass ein normativer Satz aus deskriptiven Prämissen gefolgert wird. Das verträgt sich schlecht mit der bekannten Sein-SollensThese, wonach sich keine normativen Sätzen aus rein deskriptiven ableiten lassen. ${ }^{33}$ Und in der Tat denke ich, dass der genannte Schluss eine normative Prämisse voraussetzt. Wie ich deutlich machen möchte, geht es dabei um ein Sollen, das wunschunabhängig ist.

Stellen wir uns - analog zu Stemmers moralischem Skeptiker - einen Skeptiker vor, der A möchte und sieht, dass Y das geeignete Mittel für A ist und nun fragt, wieso er Y wählen soll. Die naheliegende Antwort lautet: Weil dies eben das geeignete Mittel zur Erlangung von A ist. Das bringt den Skeptiker aber nicht zum Verstummen. Er wird weiterfragen, wieso er denn das geeignete Mittel zur Erlangung von A wählen soll. Die Antwort, weil eben, wer etwas wolle auch die geeigneten Mittel dazu wolle, wird nicht überzeugen, $\mathrm{da}$ er sich noch nicht entschieden hat, das geeignete Mittel für A zu wollen, sondern sich eben vielmehr fragt, ob er das auch wirklich wollen soll. Zudem ist es schlicht nicht richtig, dass jemand, der etwas will, eo ipso auch die dazu geeigneten Mittel will. Man kann etwas wollen, ohne auch die Mittel dazu zu wollen. ${ }^{34}$ Die richtige Antwort auf den Skeptiker lautet: „Weil man die geeigneten Mittel zur Realisierung der eigenen Wünsche wählen sollte. Und genau deshalb solltest du auch Y wählen“. Der Schluss: „Ich habe einen Grund, Y zu wählen“ (oder „Ich sollte Y wählen“) setzt die normative Prämisse, dass man die jeweils geeigneten Mittel zu seinen Zwecken wählen soll, voraus. Insofern folgt er aus folgenden Prämissen:

1. Jeder soll die geeigneten Mittel zur Realisierung deiner Wünsche wählen.

2. Ich wünsche A.

3. Y ist ein geeignetes Mittel für A.

Schluss: Ich habe einen Grund, Y zu wählen (Ich sollte Y wählen)

Dabei ist in unserem Zusammenhang folgendes von zentraler Bedeutung: Die normative Prämisse bringt ein Sollen zum Ausdruck, das auf keinem Wunsch beruht. Es ist vernünftig, die geeigneten Mittel zur Verwirklichung seiner Wünsche zu wählen, unabhängig davon, ob man dies will oder nicht. Die Handlungsgründe, die nach Stemmer allein Handlungsgründe sein können, beruhen auf einem wunschunabhängigen Sollen und entsprechend auch auf Gründen, die keine Wünsche voraussetzen. Es gäbe - anders formuliert - keine Gründe, etwas für die Realisierung seiner Wünsche zu tun, wenn es nicht Gründe gäbe, die unabhängig von irgendwelchen Wünschen bestehen würden. ${ }^{35}$

33 Vgl. dazu Hudson (ed.) (1969).

34 Wie Kant sagt, will das jemand nur dann, wenn er vernünftig ist: „Wer den Zweck will, will (so fern die Vernunft auf seine Handlung entscheidenden Einfluss hat) auch das dazu unentbehrlich notwendige Mittel, das in seiner Gewalt ist.“ Kant (1785) 417; vgl. dazu auch Korsgaard (1997) 238.

35 Vgl. dazu auch Hampton (1998) $165 f$. 
Wenn nun aber - wie Stemmer meint - wunschunabhängige Gründe mysteriöse Grössen wären, dann wären die Gründe, die wir haben, uns um unsere Wünsche zu kümmern, nicht weniger mysteriös. Wenn wir nun aber auch diese Gründe als mysteriöse Grössen zurückweisen würden, müssten wir konsequenterweise auf die Rede von Gründen verzichten und sagen, dass diese in einer aufgeklärten Moral keinen Platz hätten. Damit aber würde eine aufgeklärte Moral auf nichts anderes als auf eine Aufgabe der Idee der praktischen Normativität hinauslaufen. Das wäre wohl auch nach Stemmer ein unbefriedigendes Resultat.

\section{Schluss}

Gegen Stemmers Konzept eines sanktionsbedingten moralischen Müssen spricht, dass das moralische Müssen mit einem bloss prudentiellen Müssen zusammenfällt. Ein Begriff moralischer Verpflichtung lässt sich auf diesem Hintergrund nicht mehr aufrechterhalten. Zudem kann - anders als Stemmer glaubt - auch kein Kernbestand allgemein geltender moralischer Pflichten formuliert werden. Was Personen tun müssen, bestimmt sich nach dem, was für sie in den jeweiligen Situationen von Vorteil ist. Um dieses Problem zu lösen, darf man nicht auf innere Sanktionen hoffen, da diese in einer rationalen Moral keinen Platz haben, genausowenig wie die Disposition, sich allgemein moralisch zu verhalten. Stemmer sieht diese Schwierigkeiten, glaubt aber, dass es dazu keine Alternative gibt, sofern man keine mysteriösen Grössen, nämlich wunschunabhängige Handlungsgründe, zulassen will. Die nähere Betrachtung zeigt jedoch, dass wunschrelative Handlungsgründe, die für Stemmer die alleinigen Gründe zum Handeln sind, solche wunschunabhängigen Gründe voraussetzen. Wenn wunschunabhängige Handlungsgründe wirklich mysteriös wären, dann wären es auch die von Stemmer zugelassenen Gründe und mithin auch sein Konzept praktischer Normativität.

\section{Literatur}

Hampton, J. (1998), The Authority of Reason, Cambridge. Hudson, W. P. (ed.) (1969), The Is-Ought Question, London.

Kant, I. (1785), Grundlegung zur Metaphysik der Sitten (1785), AkademieAusgabe, Bd. IV.

Korsgaard, Ch. (1997), The Normativity of Instrumental Reason, in: G. Cullity/B. Gaut (eds.), Ethics and Practical Reason, Oxford, 215-254.

Nagel, T. (1999), Wünsche, Motive der Klugheit und die Gegenwart, in: S. Gosepath (Hg.): Motive, Gründe, Zwecke. Theorien praktischer Rationalität, Frankfurt, 146-167. 
Schaber, P. (1999), Normative Handlungsgründe, in: Analyse \& Kritik 21, 25 40.

Stemmer, P. (2000), Handeln zugunsten anderer, Berlin/New York.

Stemmer, P. (2002), Moralischer Kontraktualismus, in: Zeitschrift für philosophische Forschung 56, 1-21. 